\title{
La FMH et I'ASI soutiennent la motion sur la sécurité des patients et la garantie de la qualité dans les soins
}

\author{
Lettre aux membres du Conseil national
}

Mesdames et Messieurs,

La Fédération des médecins suisses (FMH) et l'Association suisse des infirmières et infirmiers (ASI) tiennent à exprimer leur soutien à la motion citée en titre, au moment où elle doit être discutée dans les Groupes parlementaires puis en plénum.

Comme soignants, médecins et infirmières/ infirmiers, nous tenons à redire combien nous sommes prêts à nous impliquer activement dans l'élaboration et la réalisation de projets et mesures nouveaux visant à diminuer les risques d'erreurs dans les soins et à améliorer la qualité.

La plate-forme prévue par la motion nous semble être une solution particulièrement constructive pour avancer dans la bonne direction, car la sécurité des patientes et des patients est un souci qui doit être porté conjointement par tous les participants au système de santé - y compris les soignant-es, y compris la Confédération!

Nous sommes ainsi tout à fait surpris que le Conseil fédéral considère comme «non prioritaire» la création de cette plate-forme.

Pierre Théraulaz, président ASI

Urs Weyermann, secrétaire général ASI

N. B.: Le Conseil national a traité cette motion de sa Commission de la sécurité sociale et de la santé publique en date du 3 mars 2005. Par 85 voix contre 35, il l'a transmise au Gouvernement contre la volonté de ce dernier. A ce sujet, le conseiller fédéral Pascal Couchepin a déclaré,
Les mesures «de police» suggérées par le Conseil fédéral dans sa réponse du 23 février ("prendre et renforcer les mesures nécessaires sur la base de l'art. 58 LAMal») nous semblent loin de pouvoir répondre aux besoins dans ce domaine brûlant: vouloir ainsi «ordonner» la qualité est à l'évidence une illusion qui ne peut tromper que l'administration!

Par contre, l'inscription explicite, dans la LAMal, de la sécurité des patient-es comme une responsabilité de la Confédération, et la perspective de la mise en ouvre commune, concertée, d'une réflexion puis de l'implémentation des mesures nécessaires à ce sujet, est une voie qui nous paraît pleine de promesses.

La sécurité et la qualité des soins sont un domaine par excellence où les mesures unilatérales sont vouées à l'échec, et où les possibilités de concertation doivent être soutenues par toutes et tous. La FMH et l'ASI souhaitent donc vivement que vous puissiez apporter votre soutien à cette motion de la CSSS-CN, et ils vous en remercient.

Jacques de Haller, président FMH

Yves Guisan, conseiller national, vice-président FMH

entre autres, qu'il reconnaît la nécessité de garantir la qualité des soins médicaux. Toutefois, selon lui, il n'est ni judicieux ni possible financièrement de placer cette garantie et la sécurité des patients sous la seule responsabilité de la Confédération. 